\title{
Smart Suspension System for Linear Guideways
}

\author{
Dominik Pisarski • Czeslaw I. Bajer
}

Received: 27 December 2009 / Accepted: 20 July 2010 / Published online: 7 August 2010

(C) The Author(s) 2010. This article is published with open access at Springerlink.com

\begin{abstract}
This paper presents a new method for the semi-active control of the span system of linear guideways subjected to a travelling load. Two elastic beams are coupled by a set of controlled dampers. The relative velocity of the spans provides an opportunity for efficient control via semi-active suspension. The magnitude of the moving force is assumed to be constant by neglecting inertial forces. The response of the system is solved in modal space. The full analytical solution is based on the power series method and can be given over an arbitrary time interval. The control strategy is formulated by using bilinear optimal control theory. As a result, bang-bang controls are taken into account. The final solution is obtained as a numerical mean value. Several examples demonstrate the efficiency of the proposed method. The controlled system outperforms passive solutions over a wide range. Due to the simplicity of its design, the presented solution should be interesting to engineers.
\end{abstract}

Keywords Semi-active control $•$ Smart suspension system • Vibration control • Linear guideway $\cdot$ Moving load

\section{Introduction}

The ability to make an object move along a straight or precisely controlled trajectory is essential in some technological processes such as cutting (flame, plasma,

\footnotetext{
D. Pisarski $(\varangle) \cdot$ C. I. Bajer

Institute of Fundamental Technological Research, Polish Academy of Sciences,

Pawińskiego 5b, 02-106 Warsaw, Poland

e-mail: dpisar@ippt.gov.pl

C. I. Bajer

The Faculty of Automotive and Construction Machinery Engineering, Warsaw University of Technology, Narbutta 84, 02-524 Warsaw, Poland e-mail: cbajer@ippt.gov.pl
} 
laser, textile, waterjet, glass cutting) or bonding (glueing, welding, soldering). Other areas of application especially suited to linear guideway systems are large format plotters and scanners for various industries as well as devices in the medical and semiconductor technologies. New solutions can accelerate procedures and decrease the mass and size of guideways supporting carriages.

In large-scale engineering structures like bridges or viaducts that span wide openings, beams must resist the loads due to heavy and fast vehicles. The construction of new bridges of sufficiently higher load-carrying capacity is usually limited by costs. Moreover, static strengthening can be restricted for technological reasons.

In the last decades, semi-active control methods have proven to be attractive solutions for both small and large-scale structures. Low in power consumption, controlled externally magneto or electro-rheological dampers can efficiently reduce undesired vibrations, enable a system to follow desired trajectories or increase its stability. Semi-active strategies are usually based on sky-hook and ground-hook concepts [1]. In structural control, these methods are used for the active suspension of a moving oscillator in [2,3]. In some recent papers, variable dampers are incorporated for seismic isolation, for example in $[4,5]$. A theoretical approach to the problem of controlled beam vibration damping, based on the method of optimal Lyapunov functions, is presented in [6]. In [7], the authors propose to control both parameters: stiffness and damping. The control function leads to maximum dissipation of energy. Generally, a reduction of amplitude needs to be achieved. The problem of reducing the beam vibrations using active control methods is also widely considered in the literature [8]. An actively constrained layer is applied in [9]. A beam subjected to a harmonic load was also controlled by an active method in [10]. An analysis in the frequency domain allowed the authors to reduce the maximum amplitudes. An actively controlled string system was considered in [11]. The other interesting results in structural control are presented in [12-14]. Active methods are in general more effective than semi-active ones, but the power consumption of the latter is significantly lower.

Most of the active and semi-active methods that have been developed lead to feedback controls determined by state-space measures. In the case of a continuous system, such an approach is typically complex due to observer design. The alternative method is pre-computed open loop control. This is particularly useful in problems with a well-defined excitation. In linear mechanical systems, semi-active control methods usually result in switching operations, where the parameters to be controlled (damping, stiffness) are switched between two or more values. The switching conditions are based on state or time events. Optimally switched linear systems are considered in a paper [15]. Typically, the optimal switching pattern results in a large number of switching events. If an error occurs and the switching pattern is shifted in the time domain, then such a complicated control may immediately drive the system to an undesired or even unstable state. The aim of the approach presented in this paper is to design an effective and safe switching method with reduced number of switching events.

The idea of straight-line passage is based on the principle of a two-sided lever. The first part of the beam, which is subjected to a moving load, is supported by an active damper placed on the rigid base (Fig. 1a). The first damper is active while the 


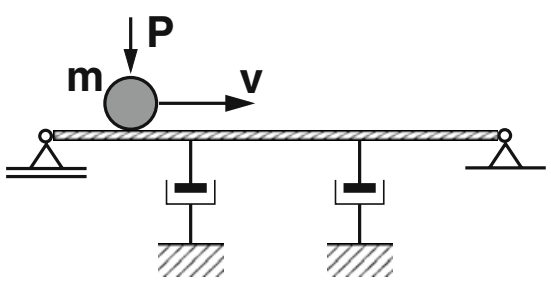

(a)

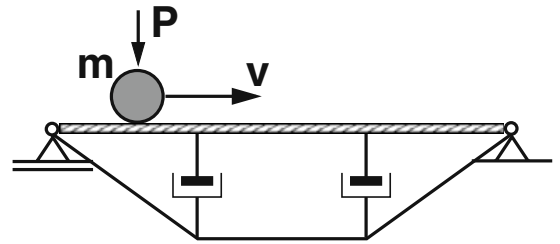

(b)

Fig. 1 Structure supported by active dampers [17]

second is passive. At this stage, part of the beam is turned about its center of gravity, levering the right-hand part with a passive damper attached. The temporal increment of displacements on the right-hand part of the beam enables us to exploit it during the second stage of the passage (Fig. 2).

Technical difficulties with the rigid support of the lower parts of our dampers require new, more practical solutions. Dampers are supported with an elastic string or bar system (Fig. 1b). However, the elastic support reduces the efficiency of performance and also involves technological problems.

In this paper, we propose a new, significantly more efficient idea, presented in Fig. 3. The main, stiff, simply-supported beam is covered by a supplementary beam, joined to the main beam by a set of controlled dampers. This upper beam can be considered to be simply supported as well, since this type of boundary condition can be implemented in a natural way. We assume that this upper beam is significantly less rigid than the main lower beam. We must emphasize here that the desired dynamic
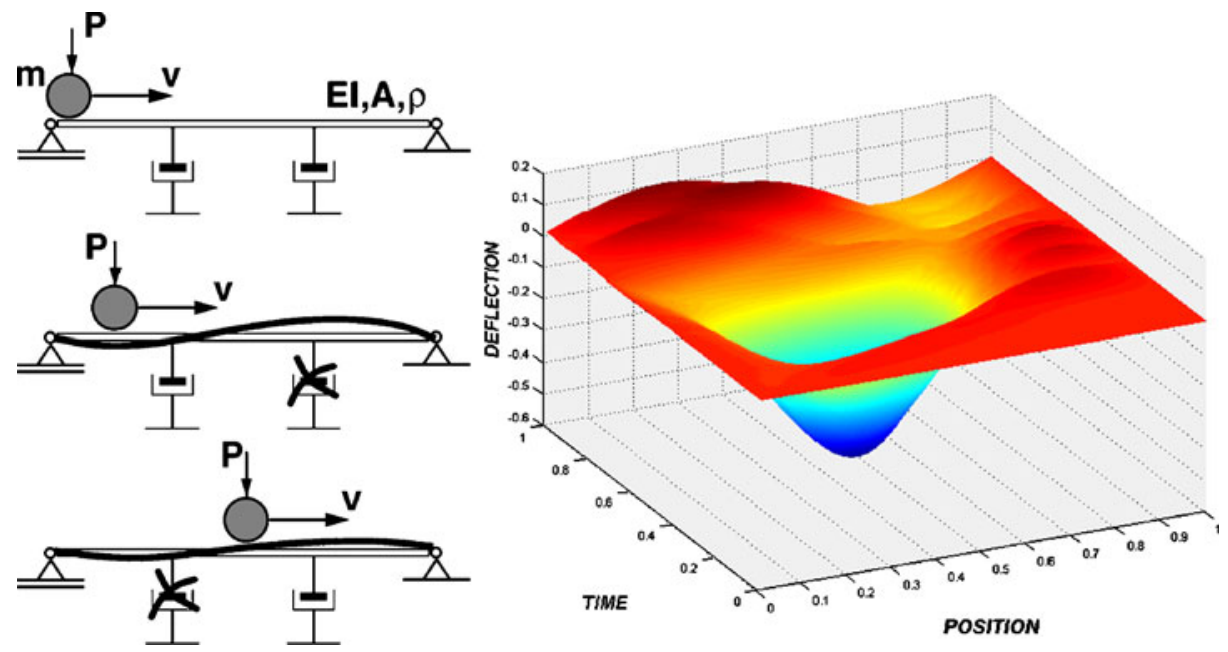

Fig. 2 The idea of semi-active control 
Fig. 3 The new idea of semi-active control of coupled beams
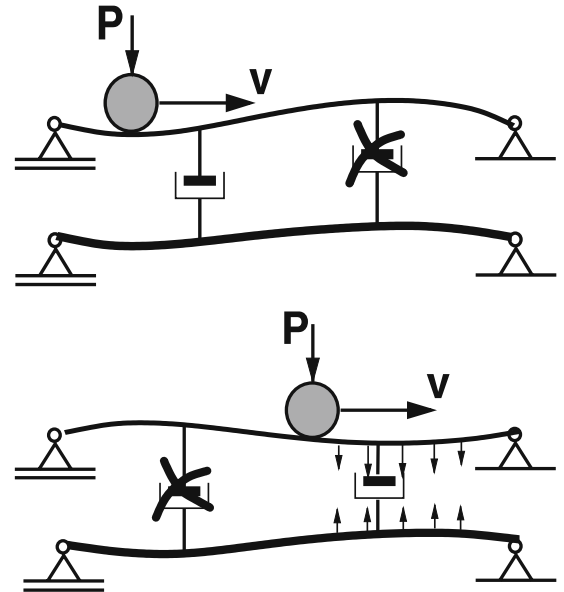

effect is obtained from the relative velocity between lower and upper beams. Let us consider the second stage of the motion depicted in Fig. 3. The upper beam when subjected to a force $P$ is deflected. At the same time, the velocity of the lower beam levers the joining damper and effectively supports the upper beam. The dynamic response of a double-beam system traversed by a constant moving load is studied in [16]. The authors explore the effects of the moving speed of the load and of the damping and stiffness of the viscoelastic layer on the deflections of the beams.

An early version of this approach was presented in [17]. The span was supported by a set of dampers placed on the rigid base. Open loop control of damping parameters allowed us to actively reduce the deflection of a string or beam supporting the travelling load. The control of beam vibrations exhibited a significantly higher control efficiency than in the case of a string.

The novelty of this approach is a modification of the main structure of the span carrying the load by a supplementary, relatively soft beam, joined to the main structure by controlled viscous elements (Fig. 4). Such a modification does not require a rigid base and can be easily incorporated into existing guideways. The relative velocity of the lower and upper beams enables us to design an efficient control for straight line passage.

Fig. 4 Semi-active linear guideway

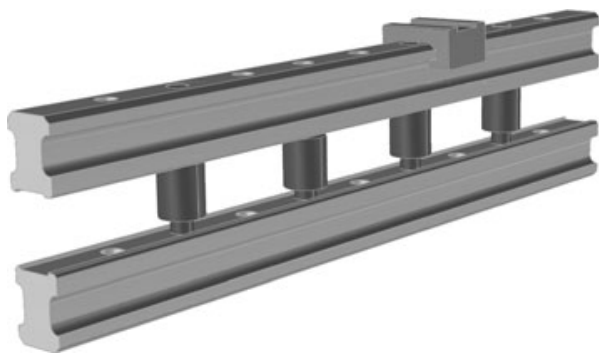


The paper is organized as follows. In Section 2, the mathematical model of the problem under consideration is given. In Section 3, the control problem is formulated and a sub-optimal solution is proposed. In Section 4, the results of numerical simulations are provided to verify the proposed method.

\section{Equations of Motion}

We consider the system shown in Fig. 5. The Bernoulli-Euler beams are simply supported and coupled by a set of control dampers. The lower beam is rigid and is considered to be the main span while the upper beam is added to increase the total load carrying capacity and is relatively soft. The magnitude of the moving force is taken as constant by neglecting the inertial forces. This is under the assumption that the mass accompanying the travelling load is small compared to the mass of the beam. The action of the massless dampers is proportional to the relative velocities of displacement at given points.

For such a system, we can write the equations of motion as follows:

$$
\begin{aligned}
& E I_{1} \frac{\partial^{4} w_{1}(x, t)}{\partial x^{4}}+\mu_{1} \frac{\partial^{2} w_{1}(x, t)}{\partial t^{2}}=-\sum_{i=1}^{m} u_{i}(t)\left[\frac{\partial w_{1}(x, t)}{\partial t}-\frac{\partial w_{2}(x, t)}{\partial t}\right] \delta\left(x-a_{i}\right) \\
&+P \delta(x-v t), \\
& E I_{2} \frac{\partial^{4} w_{2}(x, t)}{\partial x^{4}}+\mu_{2} \frac{\partial^{2} w_{2}(x, t)}{\partial t^{2}}=-\sum_{i=1}^{m} u_{i}(t)\left[\frac{\partial w_{2}(x, t)}{\partial t}-\frac{\partial w_{1}(x, t)}{\partial t}\right] \delta\left(x-a_{i}\right),
\end{aligned}
$$

together with boundary and initial conditions:

$$
\begin{aligned}
& w_{1}(0, t)=0, \quad w_{1}(l, t)=0, \quad w_{1}(x, 0)=0, \quad \dot{w}_{1}(x, 0)=0, \\
& w_{2}(0, t)=0, \quad w_{2}(l, t)=0, \quad w_{2}(x, 0)=0, \quad \dot{w}_{2}(x, 0)=0 .
\end{aligned}
$$

Here, $w_{1}(x, t)$ and $w_{2}(x, t)$ are the transverse deflections of the beams at point $(x, t)$, $u_{i}(t)$ is the $i$-th damping coefficient as a function of time, $a_{i}$ is the $i$-th fixed point of a damper, $m$ is the number of viscous supports, $\mu_{1}$ and $\mu_{2}$ are constant mass densities per unit length, $E I_{1}$ and $E I_{2}$ are the beam bending stiffnesses, $P$ is the concentrated

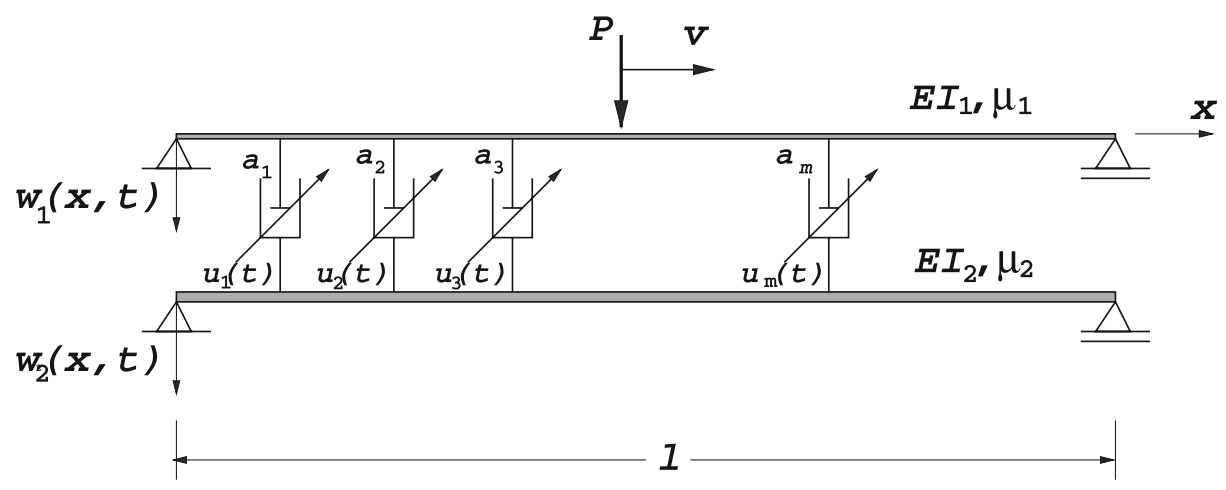

Fig. 5 Double Euler-Bernoulli beam system coupled by a set of active dampers 
force passing over the upper beam at constant velocity $v$ and $\delta$ is the Dirac delta function.

For the control design presented in the next section, it is convenient to transform the equations of motion given in PDE form (Eq. 1) into a system of ODEs. For this purpose, we apply Fourier expansions to $w_{1}(x, t)$ and $w_{2}(x, t)$ as follows:

$$
w_{1}(x, t)=\frac{2}{l} \sum_{j=1}^{\infty} V_{1}(j, t) \sin \frac{j \pi x}{l}, \quad w_{2}(x, t)=\frac{2}{l} \sum_{j=1}^{\infty} V_{2}(j, t) \sin \frac{j \pi x}{l} .
$$

Here, $\sin \frac{j \pi x}{l}=: \theta_{j}(x)$ are eigenfunctions which respect the boundary conditions (Eq. 2) and $V_{1}(j, t), V_{2}(j, t)$ are functions to be determined. The pairs $w_{1}(x, t), V_{1}(j, t)$ and $w_{2}(x, t), V_{2}(j, t)$ satisfy the relations:

$$
V_{1}(j, t)=\int_{0}^{l} w_{1}(x, t) \theta_{j}(x) \mathrm{d} x, \quad V_{2}(j, t)=\int_{0}^{l} w_{2}(x, t) \theta_{j}(x) \mathrm{d} x .
$$

Inserting Eqs. 3 into 1, we obtain:

$$
\begin{aligned}
& E I_{1} \frac{\partial^{4}}{\partial x^{4}}\left[\frac{2}{l} \sum_{j=1}^{\infty} V_{1}(j, t) \theta_{j}(x)\right]+\mu_{1} \frac{\partial^{2}}{\partial t^{2}}\left[\frac{2}{l} \sum_{j=1}^{\infty} V_{1}(j, t) \theta_{j}(x)\right] \\
& =P \delta(x-v t)-\sum_{i=1}^{m} u_{i}(t)\left\{\frac{\partial}{\partial t}\left[\frac{2}{l} \sum_{j=1}^{\infty} V_{1}(j, t) \theta_{j}(x)\right]-\frac{\partial}{\partial t}\left[\frac{2}{l} \sum_{j=1}^{\infty} V_{2}(j, t) \theta_{j}(x)\right]\right\} \\
& \qquad I_{2} \frac{\partial^{4}}{\partial x^{4}}\left[\frac{2}{l} \sum_{j=1}^{\infty} V_{2}(j, t) \theta_{j}(x)\right]+\mu_{2} \frac{\partial^{2}}{\partial t^{2}}\left[\frac{2}{l} \sum_{j=1}^{\infty} V_{2}(j, t) \theta_{j}(x)\right] \\
& =-\sum_{i=1}^{m} u_{i}(t)\left\{\frac{\partial}{\partial t}\left[\frac{2}{l} \sum_{j=1}^{\infty} V_{2}(j, t) \theta_{j}(x)\right]-\frac{\partial}{\partial t}\left[\frac{2}{l} \sum_{j=1}^{\infty} V_{1}(j, t) \theta_{j}(x)\right]\right\} \delta\left(x-a_{i}\right) .
\end{aligned}
$$

Next, each term of Eq. 5 is multiplied by $\sin \frac{k \pi x}{l}=: \theta_{k}(x)$ and then integrated with respect to $x$ over the interval $[0, l]$. This yields:

$$
\begin{aligned}
& E I_{1}\left(\frac{j \pi}{l}\right)^{4}\left[\frac{2}{l} \sum_{j=1}^{\infty} V_{1}(j, t) \int_{0}^{l} \theta_{j}(x) \theta_{k}(x) \mathrm{d} x\right]+\mu_{1}\left[\frac{2}{l} \sum_{j=1}^{\infty} \ddot{V}_{1}(j, t) \int_{0}^{l} \theta_{j}(x) \theta_{k}(x) \mathrm{d} x\right] \\
& =-\sum_{i=1}^{m} u_{i}(t)\left[\frac{2}{l} \sum_{j=1}^{\infty}\left[\dot{V}_{1}(j, t)-\dot{V}_{2}(j, t)\right] \int_{0}^{l} \theta_{j}(x) \theta_{k}(x) \delta\left(x-a_{i}\right) \mathrm{d} x\right] \\
& +P \int_{0}^{l} \theta_{k}(x) \delta(x-v t) \mathrm{d} x, \\
& E I_{2}\left(\frac{j \pi}{l}\right)^{4}\left[\frac{2}{l} \sum_{j=1}^{\infty} V_{2}(j, t) \int_{0}^{l} \theta_{j}(x) \theta_{k}(x) \mathrm{d} x\right]+\mu_{2}\left[\frac{2}{l} \sum_{j=1}^{\infty} \ddot{V}_{2}(j, t) \int_{0}^{l} \theta_{j}(x) \theta_{k}(x) \mathrm{d} x\right] \\
& =-\sum_{i=1}^{m} u_{i}(t)\left[\frac{2}{l} \sum_{j=1}^{\infty}\left[\dot{V}_{2}(j, t)-\dot{V}_{1}(j, t)\right] \int_{0}^{l} \theta_{j}(x) \theta_{k}(x) \delta\left(x-a_{i}\right) \mathrm{d} x\right] .
\end{aligned}
$$


Equations 6 are fulfilled for $\mathrm{k}=1,2, \ldots$ The eigenfunctions $\theta_{j}(x), \theta_{k}(x)$ satisfy the orthogonality conditions:

$$
\int_{0}^{l} \theta_{j}(x) \theta_{k}(x) \mathrm{d} x=\frac{l}{2} \delta_{j, k},
$$

where $\delta_{j, k}$ is the Kronecker delta. The terms where the Dirac delta is incorporated are computed below:

$$
\begin{aligned}
\int_{0}^{l} \theta_{j}(x) \theta_{k}(x) \delta\left(x-a_{i}\right) \mathrm{d} x & =\theta_{j}\left(a_{i}\right) \theta_{k}\left(a_{i}\right), \\
\int_{0}^{l} \theta_{k}(x) \delta(x-v t) & =\theta_{k}(v t) .
\end{aligned}
$$

Thus, Eq. 6 can be written as:

$$
\begin{aligned}
\mu_{1} \sum_{j=1}^{\infty} \ddot{V}_{1}(j, t) \delta_{j, k} & +\frac{2}{l} \sum_{i=1}^{m} u_{i}(t) \sum_{j=1}^{\infty}\left[\dot{V}_{1}(j, t)-\dot{V}_{2}(j, t)\right] \theta_{j}\left(a_{i}\right) \theta_{k}\left(a_{i}\right) \\
& +E I_{1} \sum_{j=1}^{\infty}\left(\frac{j \pi}{l}\right)^{4} V_{1}(j, t) \delta_{j, k}=P \theta_{k}(v t), \\
\mu_{2} \sum_{j=1}^{\infty} \ddot{V}_{2}(j, t) \delta_{j, k} & +\frac{2}{l} \sum_{i=1}^{m} u_{i}(t) \sum_{j=1}^{\infty}\left[\dot{V}_{2}(j, t)-\dot{V}_{1}(j, t)\right] \theta_{j}\left(a_{i}\right) \theta_{k}\left(a_{i}\right) \\
& +E I_{2} \sum_{j=1}^{\infty}\left(\frac{j \pi}{l}\right)^{4} V_{2}(j, t) \delta_{j, k}=0, \quad k=1,2, \ldots
\end{aligned}
$$

Finally we write PDE (Eq. 1) as a system of ODEs:

$$
\begin{aligned}
\mu_{1} \ddot{V}_{1}(k, t) & +\frac{2}{l} \sum_{i=1}^{m} \sum_{j=1}^{\infty} u_{i}(t)\left[\dot{V}_{1}(j, t)-\dot{V}_{2}(j, t)\right] \sin \frac{j \pi a_{i}}{l} \sin \frac{k \pi a_{i}}{l} \\
& +E I_{1} \frac{k^{4} \pi^{4}}{l^{4}} V_{1}(k, t)=P \sin \frac{k \pi v t}{l}, \\
\mu_{2} \ddot{V}_{2}(k, t) & +\frac{2}{l} \sum_{i=1}^{m} \sum_{j=1}^{\infty} u_{i}(t)\left[\dot{V}_{2}(j, t)-\dot{V}_{1}(j, t)\right] \sin \frac{j \pi a_{i}}{l} \sin \frac{k \pi a_{i}}{l} \\
& +E I_{2} \frac{k^{4} \pi^{4}}{l^{4}} V_{2}(k, t)=0, \quad k=1,2, \ldots .
\end{aligned}
$$

Furthermore, we consider only approximate solutions of Eqs. 1, 2 by using a finitedimensional modal space i.e. $j, k=1,2, \ldots, M<\infty$.

\section{Formulation of Control Problem}

The control method presented in this paper is based on the classical Pontryagin Maximum Principle [18]. We consider the optimal control problem where 
$\mathbf{y}(t) \in \mathbb{R}^{n}\left(y_{4 k-3}(t)=V_{1}(k, t), y_{4 k-2}(t)=\dot{V}_{1}(k, t), y_{4 k-1}(t)=V_{2}(k, t), y_{4 k}(t)=\dot{V}_{2}(k, t)\right.$, $k=1,2, \ldots, n / 4=M)$ denotes the generalized state vector and $\mathbf{u}(t) \in \mathbb{R}^{m}$ denotes the control variable in the time interval $\left[0, t_{f}\right]$ with final time $t_{f}=l / v$ :

$$
\begin{gathered}
\text { Minimize } J=\int_{0}^{t_{f}}\left[\sum_{k=1}^{n / 4} y_{4 k-3}(t) \sin \frac{k \pi v t}{l}\right]^{2} \mathrm{~d} t, \\
\text { subject to } \dot{\mathbf{y}}(t)=\mathbf{A} \mathbf{y}(t)+\sum_{i=1}^{m} \mathbf{B}_{i} \mathbf{y}(t) u_{i}(t)+\mathbf{f}(t), \\
y_{4 k-3}(0)=V_{1}(k, 0), \quad y_{4 k-2}(0)=\dot{V}_{1}(k, 0), \\
y_{4 k-1}(0)=V_{2}(k, 0), \quad y_{4 k}(0)=\dot{V}_{2}(k, 0), \quad k=1,2, \ldots, n / 4, \\
u_{i}(t) \in\left[0, u_{\max }\right], \quad \forall t \in\left[0, t_{f}\right], \quad i=1,2, \ldots, m .
\end{gathered}
$$

Here, $\mathbf{A}, \mathbf{B}_{i=1,2, \ldots, m} \in \mathbb{R}^{n \times n}$ are constant matrices and $\mathbf{f}(t) \in \mathbb{R}^{n}$ is an excitation vector. The cost integrand (Eq. 11) is defined in such a way that its value is related to the total deflection of the travelling load trajectory. Searching for the minimum of $J$ is equivalent to pursuing the straightest line motion of the carriage over the guideway.

Equation 12 represents a non-autonomous bilinear system (BLS) that involves the product of state and control. Due to its numerous engineering applications, the optimal control of BLS is widely studied in the literature [19,20]. Introducing a new state variable $\dot{y}_{n+1}(t)=1, y_{n+1}(0)=0$ and rebuilding $\mathbf{A} \longrightarrow \tilde{\mathbf{A}}, \mathbf{B}_{i} \longrightarrow \tilde{\mathbf{B}}_{i}(i=1,2, \ldots, m)$, $\mathbf{f}(t) \longrightarrow \tilde{\mathbf{f}}(\mathbf{y})$ in such a way they respect a new variable, we replace Eqs. 11-14 with the autonomous optimal control problem and the Maximum Principle can be applied directly. The Hamiltonian function is given by

$$
H(\mathbf{y}, \mathbf{u}, \boldsymbol{\eta})=\langle\boldsymbol{\eta}, \tilde{\mathbf{A}} \mathbf{y}\rangle+\sum_{i=1}^{m}\left\langle\boldsymbol{\eta}, \tilde{\mathbf{B}}_{i} \mathbf{y}\right\rangle u_{i}+\langle\boldsymbol{\eta}, \tilde{\mathbf{f}}\rangle-\left[\sum_{k=1}^{n / 4} y_{4 k-3}(t) \sin \frac{k \pi v y_{n+1}}{l}\right]^{2},
$$

where the vector $\eta(t) \in \mathbb{R}^{n+1}$ is the adjoint variable. The adjoint differential equation and transversality conditions are as follows:

$$
\dot{\eta}(t)=-\frac{\partial H}{\partial \mathbf{y}}, \quad \eta\left(t_{f}\right)=0 .
$$

The Hamiltonian (Eq. 15) takes a maximum value when the controls equal:

$$
u_{i}(t)=\left\{\begin{array}{ll}
u_{\max }, & \left\langle\boldsymbol{\eta}(t), \tilde{\mathbf{B}}_{i} \mathbf{y}(t)\right\rangle>0 \\
0, & \left\langle\boldsymbol{\eta}(t), \tilde{\mathbf{B}}_{i} \mathbf{y}(t)\right\rangle<0
\end{array} .\right.
$$

Hence, the optimal controls are bang-bang type controls. This result was predicted by means of numerical simulation in the former work of the authors [17]. Unfortunately, the solution of Eq. 17 is given in implicit form. Since a multidimensional problem is considered, only appropriate numerical methods are relevant. The fundamental idea behind these methods lies in transforming the optimal problem in such a way that 
the switching times of the assumed control are considered to be new parameters. The cost functional is optimised with respect to these values. So, in fact, the optimal control problem becomes a nonlinear programming problem, where non-gradient optimisation methods can be applied.

According to this approach, we assume one switching action for every control. The proposed strategy comes from numerous simulations and its basic idea is shown in Fig. 3. The dampers placed on the left-hand side are first set on, then after a certain time they are switched into the off state. The situation for the rest of the dampers is reversed. Formally, this can be written as follows:

$$
\begin{gathered}
u_{i}(t)=u_{\max } \mathcal{U}(t)-u_{\max } \mathcal{U}\left(t-\tau_{i}\right), \quad i=1,2, \ldots, m^{\prime}, \\
u_{i}(t)=u_{\max } \mathcal{U}\left(t-\tau_{i}\right), \quad i=m^{\prime}+1, m^{\prime}+2, \ldots, m,
\end{gathered}
$$

where $\tau_{i}$ is the switching time of the $i$-th damper and $\mathcal{U}(t)$ is a unit step function. The position of the damper with index $m^{\prime}$ can be assumed after preliminary numerical simulations. The optimal switching times are the solutions of the problem:

$$
\left(\tau_{1}, \tau_{2}, \ldots, \tau_{m}\right)=\underset{\tau_{1}, \tau_{2}, \ldots, \tau_{m} \in\left(0, t_{f}\right]}{\arg \min } J(\mathbf{y}(t))
$$

where $\mathbf{y}(t)$ is the resulting trajectory under controls (Eq. 18).

To obtain the trajectories $\mathbf{y}(t)$ (used in the non-gradient optimising method), we propose the solution of the system of ODEs (Eq. 10) by using power expansions for $V_{1}(k, t)$ and $V_{2}(k, t)$ calculated in the time domain split into intervals that are bounded by every pair of switching events:

$$
\begin{aligned}
& V_{1}(k, t)=\sum_{n=0}^{M} d_{n}(1, k)(t-\hat{\tau})^{n}, \\
& V_{2}(k, t)=\sum_{n=0}^{M} d_{n}(2, k)(t-\hat{\tau})^{n} .
\end{aligned}
$$

Here, $\hat{\tau}$ is first equal to zero, and then $\hat{\tau} \in\left(0, t_{f}\right]$ are the times of successive switching events. The time marching scheme allows us to proceed to successive layers with initial conditions taken from the end of previous stages.

Substitution of Eqs. 20 into 10, after some simple algebraic transformations, yields the system of recurrence equations for sequences $d_{n}(1, k)$ and $d_{n}(2, k)$ :

$$
\begin{aligned}
\mu_{1}(2 n+1)(2 n+2) d_{2 n+2}(1, k)= & -E I_{1} \frac{k^{4} \pi^{4}}{l^{4}} d_{2 n}(1, k)-\frac{2}{l} \sum_{i=1}^{m} \sum_{j=1}^{M} u_{i}(t) \alpha_{i j k}(2 n+1) \\
& \times\left[d_{2 n+1}(1, j)-d_{2 n+1}(2, j)\right] \\
& +P \sin (k \omega \hat{\tau}) \frac{(-1)^{n}(k \omega)^{2 n}}{(2 n) !}, \\
\mu_{2}(2 n+1)(2 n+2) d_{2 n+2}(2, k)= & -E I_{2} \frac{k^{4} \pi^{4}}{l^{4}} d_{2 n}(2, k)-\frac{2}{l} \sum_{i=1}^{m} \sum_{j=1}^{M} u_{i}(t) \alpha_{i j k}(2 n+1) \\
& \times\left[d_{2 n+1}(2, j)-d_{2 n+1}(1, j)\right]
\end{aligned}
$$




$$
\begin{aligned}
\mu_{1}(2 n+2)(2 n+3) d_{2 n+3}(1, k)= & -E I_{1} \frac{k^{4} \pi^{4}}{l^{4}} d_{2 n+1}(1, k)-\frac{2}{l} \sum_{i=1}^{m} \sum_{j=1}^{M} u_{i}(t) \alpha_{i j k}(2 n+2) \\
& \times\left[d_{2 n+2}(1, j)-d_{2 n+2}(2, j)\right] \\
& +P \cos (k \omega \hat{\tau}) \frac{(-1)^{n}(k \omega)^{2 n+1}}{(2 n+1) !}, \\
\mu_{2}(2 n+2)(2 n+3) d_{2 n+3}(2, k)= & -E I_{2} \frac{k^{4} \pi^{4}}{l^{4}} d_{2 n+1}(2, k)-\frac{2}{l} \sum_{i=1}^{m} \sum_{j=1}^{M} u_{i}(t) \alpha_{i j k}(2 n+2) \\
& \times\left[d_{2 n+2}(2, j)-d_{2 n+2}(1, j)\right],
\end{aligned}
$$

where the following notation is introduced: $\pi v / l=\omega, \sin \left(j \pi a_{i} / l\right) \sin \left(k \pi a_{i} / l\right)=\alpha_{i j k}$. The controls $u_{i}(t)$ are constant in every time interval as stated in Eq. 18. The first few terms of the sequences appear directly as initial conditions $d_{0}(1, j)=$ $V_{1}(j, \hat{\tau}), d_{1}(1, j)=\dot{V}_{1}(j, \hat{\tau}), d_{0}(2, j)=V_{2}(j, \hat{\tau}), d_{1}(2, j)=\dot{V}_{2}(j, \hat{\tau})$. The convergence of series (Eq. 20) is studied in [17]. There, the full derivation of Eqs. 21, 22 is also shown.

\section{Numerical Results}

In this section, the efficiency of the designed control method is verified by means of numerical simulations. Comparisons with uncontrolled cases are presented and discussed for a wide range of velocities of the moving load. Three cases are evaluated for different numbers of active dampers.

We consider the system shown in Fig. 5. Constants for the lower beam are as follows: $l=5 \mathrm{~m}, \mu_{2}=16.8 \mathrm{~kg} / \mathrm{m}, E I_{2}=2 \cdot 10^{5} \mathrm{Nm}^{2}\left(E=210 \cdot 10^{9} \mathrm{~Pa}\right)$. The upper beam, which carries the load, is treated with three different values of bending stiffness. These are given as fractions of $E I_{2}$, as follows: $E I_{1}=E I_{2} / 20$, $E I_{1}=E I_{2} / 5, E I_{1}=E I_{2} / 2$. The force $P=100 \mathrm{~N}$ travels with velocity $v=0.1 c_{\mathrm{cr}}$, $v=0.5 c_{\mathrm{cr}}, v=0.9 c_{\mathrm{cr}}$, where $c_{\mathrm{cr}}$ denotes the critical speed of the lower beam and $c_{\mathrm{cr}}=(\pi / l) \sqrt{E I_{2} / \mu_{2}}$. In the computations, the following placements of the dampers

Table 1 Cost values and optimal switching times for different speeds of the travelling load (active suspension/passive suspension), for the cases: (*) $E I_{1}=E I_{2} / 20$, (**) $E I_{1}=E I_{2} / 5$, (***) $E I_{1}=E I_{2} / 2$ with four active dampers

\begin{tabular}{ccc}
\hline Velocity & $\begin{array}{l}\text { Cost values } \\
(\text { active/passive }) \cdot 10^{-5}\end{array}$ & $\left(\tau_{1}, \tau_{2}, \tau_{3}, \tau_{4}\right) / t_{f}$ \\
\hline Case $(*)$ & & \\
$0.1 c_{\mathrm{cr}}$ & $31.079 / 61.886$ & $(0.574,0.137,0.298,0.580)$ \\
$0.5 c_{\mathrm{cr}}$ & $5.9379 / 13.492$ & $(0.622,0.185,0.420,0.553)$ \\
$0.9 c_{\mathrm{cr}}$ & $1.0308 / 4.7160$ & $(0.220,0.216,0.464,0.706)$ \\
Case $(* *)$ & & \\
$0.1 c_{\mathrm{cr}}$ & $47.796 / 51.775$ & $(0.797,0.087,0.292,0.600)$ \\
$0.5 c_{\mathrm{cr}}$ & $9.2959 / 11.279$ & $(0.837,0.168,0.338,0.566)$ \\
$0.9 c_{\mathrm{cr}}$ & $2.6669 / 3.7560$ & $(0.860,0.203,0.464,0.533)$ \\
Case $(* * *)$ & & \\
$0.1 c_{\mathrm{cr}}$ & $38.659 / 39.748$ & $(0.886,0.069,0.226,0.400)$ \\
$0.5 c_{\mathrm{cr}}$ & $8.5706 / 9.0609$ & $(0.854,0.140,0.293,0.466)$ \\
$0.9 c_{\mathrm{cr}}$ & $2.8183 / 3.1338$ & $(0.724,0.202,0.401,0.466)$ \\
\hline
\end{tabular}


Table 2 Cost values and optimal switching times for different speeds of the travelling load (active suspension/passive suspension), for the cases: (*) $E I_{1}=E I_{2} / 20$, (**) $E I_{1}=E I_{2} / 5$, $(* * *) E I_{1}=E I_{2} / 2$ with three active dampers

\begin{tabular}{ccc}
\hline Velocity & $\begin{array}{l}\text { Cost values } \\
(\text { active/passive }) \cdot 10^{-5}\end{array}$ & $\left(\tau_{1}, \tau_{2}, \tau_{3}\right) / t_{f}$ \\
\hline Case $(*)$ & & \\
$0.1 c_{\mathrm{cr}}$ & $43.395 / 70.854$ & $(0.802,0.137,0.382)$ \\
$0.5 c_{\mathrm{cr}}$ & $8.1419 / 14.899$ & $(0.746,0.241,0.474)$ \\
$0.9 c_{\mathrm{cr}}$ & $2.1244 / 5.8716$ & $(0.565,0.276,0.618)$ \\
Case $(* *)$ & & \\
$0.1 c_{\mathrm{cr}}$ & $50.831 / 55.766$ & $(0.729,0.256,0.498)$ \\
$0.5 c_{\mathrm{cr}}$ & $10.273 / 11.630$ & $(0.918,0.226,0.304)$ \\
$0.9 c_{\mathrm{cr}}$ & $2.9417 / 3.9570$ & $(0.860,0.203,0.464)$ \\
Case $(* * *)$ & & \\
$0.1 c_{\mathrm{cr}}$ & $40.886 / 41.970$ & $(0.442,0.072,0.270)$ \\
$0.5 c_{\mathrm{cr}}$ & $8.8617 / 9.2059$ & $(0.788,0.252,0.452)$ \\
$0.9 c_{\mathrm{cr}}$ & $2.9621 / 3.2004$ & $(0.892,0.209,0.416)$ \\
\hline
\end{tabular}

were established: $[0.2 l, 0.4,0.6 l, 0.8 l] ;[0.2 l, 0.5 l, 0.8 l] ;[0.25 l, 0.75 l]$ for the cases when the number of active dampers was four, three and two, respectively.

We assume controls as follows:

$$
\begin{gathered}
u_{i}(t)=u_{\max } \mathcal{U}(t)-u_{\max } \mathcal{U}\left(t-\tau_{i}\right), \quad i=1, \\
u_{i}(t)=u_{\max } \mathcal{U}\left(t-\tau_{i}\right), \quad i=2,3,4
\end{gathered}
$$

In every case, we set the value $u_{\max }=5 \cdot 10^{4} \mathrm{Ns} / \mathrm{m}$.

For optimization, we use the Hooke-Jeeves Direct Search Method [21]. In the computations, we consider at least three different starting points with three reducing step size schemes for each case. 10 modes and 40 terms in the power series were taken into account in computations. Optimal switching time vectors and related cost values are summarized in Tables 1, 2, 3. By the passive case, we mean a constant damping $u_{i}(t)=u_{\max }, \forall t \in\left[0, t_{f}\right]$. By $w(v t, t)$ in the following figures we denote the transverse deflection under the moving load.

The best efficiency of the proposed strategy, measured as the fraction of cost values, active/passive, is obtained in the cases where $E I_{1}=E I_{2} / 20$. In case of four

Table 3 Cost values and optimal switching times for different speeds of the travelling load (active suspension/passive suspension), for the cases: (*) $E I_{1}=E I_{2} / 20$, (**) $E I_{1}=E I_{2} / 5$, (***) $E I_{1}=E I_{2} / 2$ with two active dampers

\begin{tabular}{ccc}
\hline Velocity & $\begin{array}{l}\text { Cost values } \\
(\text { active/passive }) \cdot 10^{-5}\end{array}$ & $\left(\tau_{1}, \tau_{2}\right) / t_{f}(*)$ \\
\hline Case $(*)$ & $80.687 / 99.486$ & $(0.683,0.147)$ \\
$0.1 c_{\mathrm{cr}}$ & $12.583 / 20.997$ & $(0.488,0.383)$ \\
$0.5 c_{\mathrm{cr}}$ & $4.5709 / 7.3663$ & $(0.372,0.547)$ \\
$0.9 c_{\mathrm{cr}}$ & & \\
Case $(* *)$ & $61.739 / 65.040$ & $(0.680,0.098)$ \\
$0.1 c_{\mathrm{cr}}$ & $10.877 / 12.855$ & $(0.562,0.317)$ \\
$0.5 c_{\mathrm{cr}}$ & $3.5002 / 4.7080$ & $(0.446,0.434)$ \\
$0.9 c_{\mathrm{cr}}$ & & \\
Case $(* * *)$ & $45.447 / 46.139$ & $(0.672,0.076)$ \\
$0.1 c_{\mathrm{cr}}$ & $9.1654 / 9.6521$ & $(1.000,0.286)$ \\
$0.5 c_{\mathrm{cr}}$ & $3.1412 / 3.4141$ & $(0.518,0.366)$ \\
$0.9 c_{\mathrm{cr}}$ & & \\
\hline
\end{tabular}



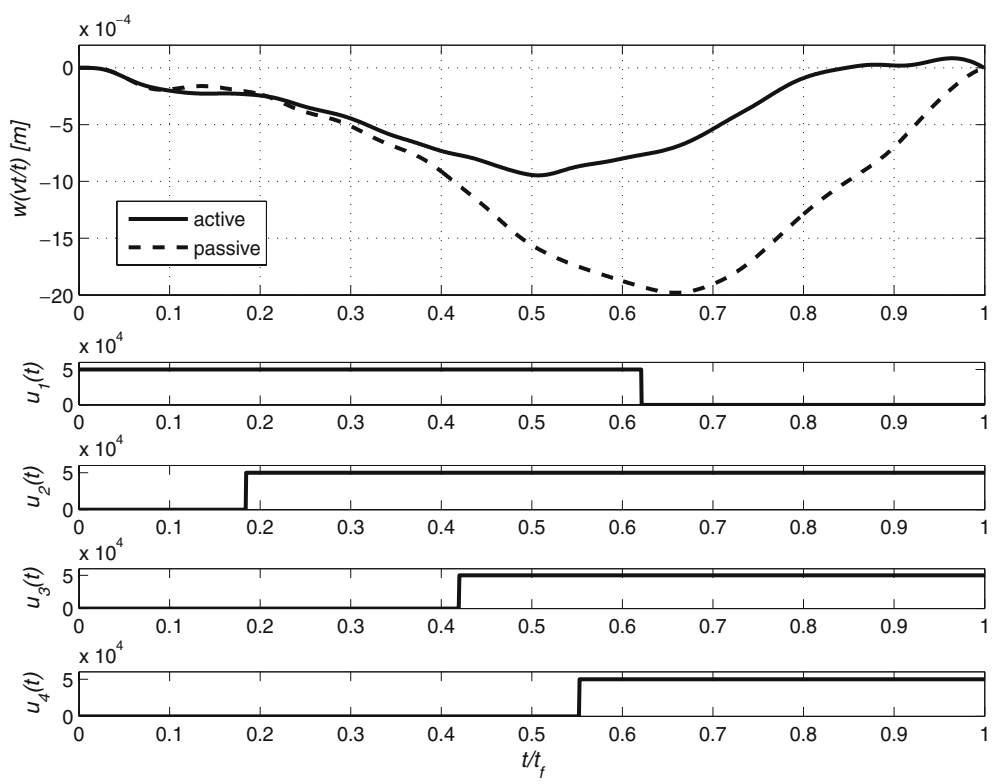

Fig. 6 Extremal deflection trajectory and controls in the case $E I_{1} / E I_{2}=1 / 20, v=0.5 c_{\text {cr }}$ with four active dampers
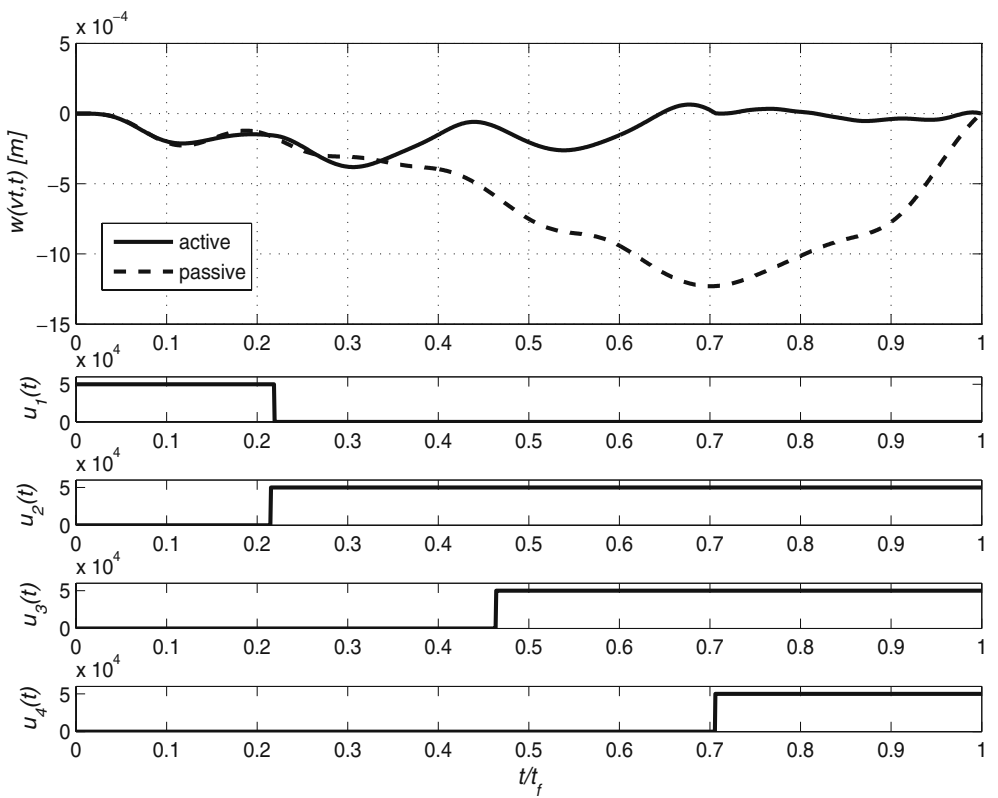

Fig. 7 Extremal deflection trajectory and controls in the case $E I_{1} / E I_{2}=1 / 20, v=0.9 c_{\text {cr }}$ with four active dampers 


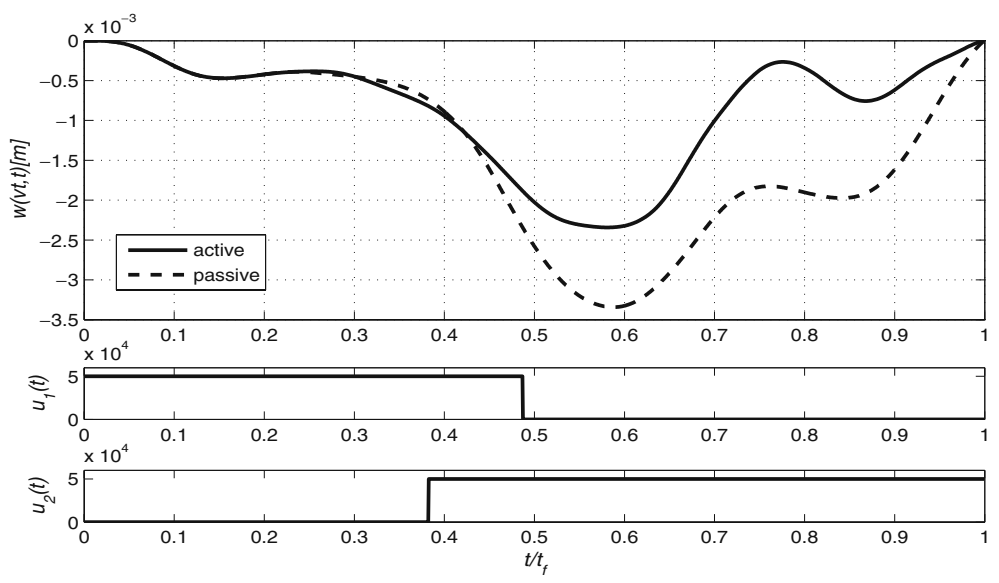

Fig. 8 Extremal deflection trajectory and controls in the case $E I_{1} / E I_{2}=1 / 20, v=0.5 c_{\text {cr }}$ with two active dampers

active dampers, for the carriage travelling at high speed $v=0.5 c_{\mathrm{cr}}, v=0.9 c_{\mathrm{cr}}$ the maximum deflection was reduced by a factor of almost two (Fig. 6) and three (Fig. 7), respectively. The latter trajectory is almost flat for more than half of the travel time. For carriage movement at low speed, the dynamic effects are observed to be weak and the controls cannot change the process efficiently.

For a lower number of active dampers, we observe an increasing deflection near the position of the absent damper (Fig. 8). To provide a flat trajectory in the second stage of the passage, at least two active dampers have to be placed on the right-hand part of the beam to support the travelling load (Fig. 9).
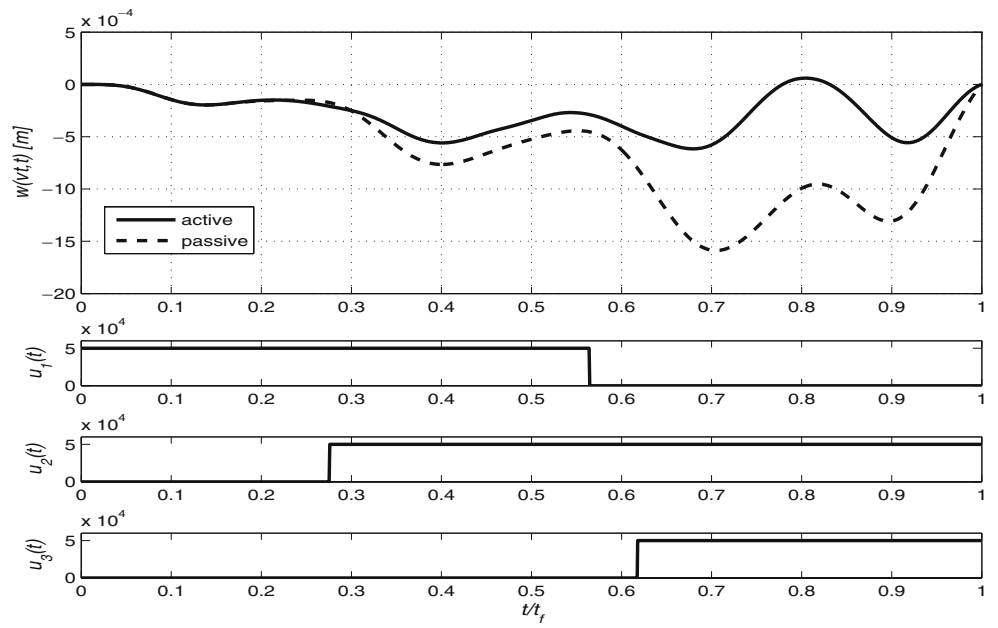

Fig. 9 Extremal deflection trajectory and controls in the case $E I_{1} / E I_{2}=1 / 20, v=0.9 c_{\mathrm{cr}}$ with three active dampers 
Table 4 Cost values and optimal switching times for different placement of dampers (active suspension/passive suspension), for the case $E I_{1}=E I_{2} / 20,0.9 c_{\mathrm{cr}}$ with two active dampers

\begin{tabular}{lll}
\hline Placement of dampers & Cost values (active/passive) $\cdot 10^{-5}$ & $\left(\tau_{1}, \tau_{2}\right) / t_{f}$ \\
\hline$[0.3 l, 0.63 l]$ & $5.1557 / 8.5831$ & $(0.350,0.412)$ \\
{$[0.3333 l, 0.6666 l]$} & $5.1312 / 8.7453$ & $(0.379,0.465)$ \\
{$[0.36 l, 0.69 l]$} & $4.8080 / 8.6058$ & $(0.540,0.520)$ \\
\hline
\end{tabular}

To demonstrate how the placement of dampers affects the control efficiency, we compare the following three cases: $[0.3 l, 0.63 l]$; $[0.3333 l, 0.6666 l] ;[0.36 l, 0.69 l]$. The results are summarized in Table 4. The comparison of passive cases are presented in Fig. 10. There is no control action on the third mode in the second case (see Eq. 10). It seems intuitive that the best control capability is achieved when $\sin \frac{k \pi a_{i}}{l} \neq 0$ for the first few modes i.e. $k=1,2,3$. However, in some cases the better effect could be obtained by letting a certain mode to stay out of the suspension to increase its velocity. Then it might beneficially affect other modes by the higher rate of damping force. The complete analysis how the placement of dampers affects the control efficiency needs further detailed study. Its high complication rate is associated with conjugate structure of ODEs that describe the physical system. More extensive investigation is addressed in future works.

To show how the proposed system corresponds to a simple guideway, represented by a traditional single-beam span, we compare the trajectories of a carriage travelling along the controlled system and along a single beam with increased bending stiffness $E I_{2}, 2 \cdot E I_{2}, 4 \cdot E I_{2}, 8 \cdot E I_{2}$ (Fig. 11). In this case, we obtain a relatively flat trajectory if we increase the stiffness parameter by more than eight times. At the same time, it requires an increased mass for the guideway and directly affects the static deflection curve.

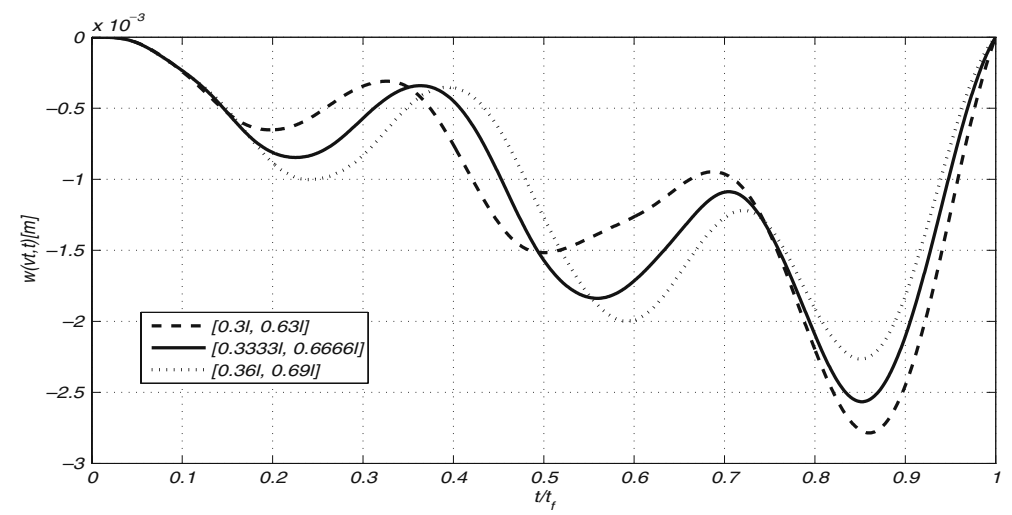

Fig. 10 Deflection trajectories for different placement of dampers for the case $E I_{1} / E I_{2}=1 / 20$, $v=0.9 c_{\text {cr }}$ with two passive dampers 


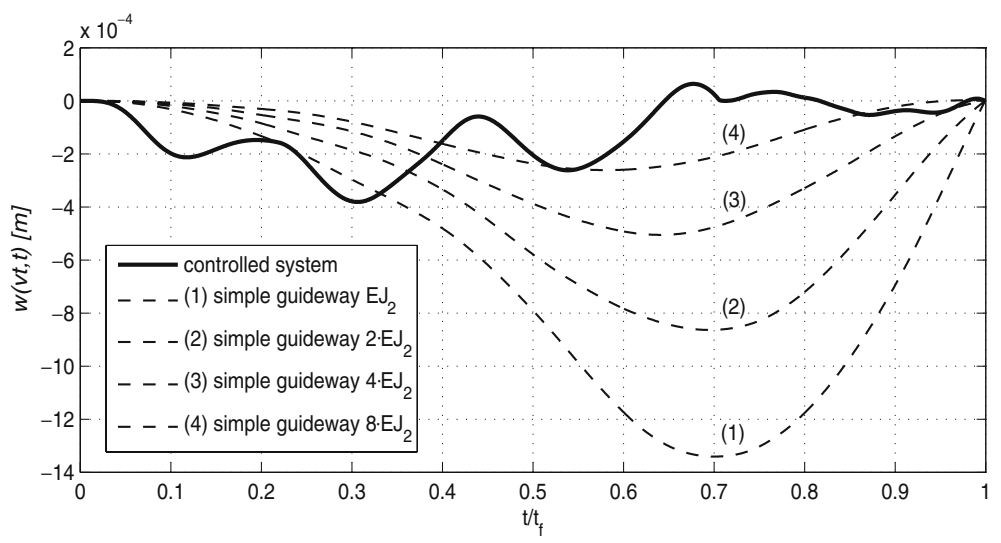

Fig. 11 Carriage trajectories when travelling over controlled system and simple guideways, $v=0.9 c_{\mathrm{cr}}$ with four active dampers

\section{Conclusions}

In this paper, a semi-active control method for linear guideways has been presented. This work is an extension of an earlier approach proposed by the authors in paper [17]. The new idea of a semi-active double beam system does not require the presence of a rigid base. The performance of a bang-bang control method has been verified for different system parameters and different number of active dampers. The placement of dampers significantly affects the shape of the carriage trajectory. The best efficiency of the proposed control method is obtained at high travel speeds. The controlled system can efficiently reduce the mass of the guideway. The control strategy is simple for a practical design. It can be implemented by creating an optimal control map in the memory of the controller. Integration of a neural network with the system will be addressed in future works.

Open Access This article is distributed under the terms of the Creative Commons Attribution Noncommercial License which permits any noncommercial use, distribution, and reproduction in any medium, provided the original author(s) and source are credited.

\section{References}

1. Sapinski, R., Pilat, A.: Semi-active suspension with a MR damper. Mach. Dyn. Probl. 27(2), 125-134 (1998)

2. Giraldo, D., Dyke, Sh.J.: Control of an elastic continuum when traversed by a moving oscillator. Struct. Control Health Monit. 14, 197-217 (2007)

3. Chen, Y., Tan, C.A., Bergman, L.A., Tsao, T.C.: Smart suspension systems for bridge-friendly vehicles. In: SPIE Proceedings Series, vol. 4696, pp. 52-61 (2002)

4. Ruangrassamee, A., Kawashima, K.: Control of nonlinear bridge response with pounding effect by variable dampers. Eng. Struct. 25, 593-606 (2003)

5. Yoshida, K., Fujio, T.: Semi-active base isolation for a building structure. Int. J. Comput. Appl. Technol. 13, 52-58 (2000) 
6. Ossowski, A.: Semi-active control of free beam vibration. Theoret. Foundations of Civil Eng. 11, 557-566 (2003)

7. Fulin, Z., Ping, T., Weiming, Y., Lushun, W.: Theoretical and experimental research on a new system of semi-active structural control with variable stiffness and damping. Earthquake Engineering and Engineering Vibration 1, 130-135 (2002)

8. Frischgesel, T., Popp, K., Reckmann, H., Schütte, O.: Regelung eines elastischen Fahrwegs inter Verwendung eines variablen Beobachters. Tech. Mech. 18(1), 44-55 (1998)

9. Baz, A.: Dynamic boundary control of beams using active constrained layer damping. Mech. Syst. Signal Process. 11(6), 811-825 (1997)

10. Pietrzakowski, M.: Active damping of beams by piezoelectric system: effects of bonding layer properties. Int. J. Solids Struct. 38, 7885-7897 (2001)

11. Tan, C.A., Ying, S.: Active wave control of the axially moving string. J. Sound Vib. 236(5), 861-880 (2000)

12. Soong, T.: Active Hybrid and Semi-active Strucural Control. Wiley, West Sussex (2005)

13. Wang, J.F., Lin, C.C., Chen, B.L.: Vibration suppression for high-speed railway bridges using tuned mass dampers. Int. J. Solids Struct. 40, 465-491 (2003)

14. Hui, L., Wenli, C., Jinping, O.: Semiactive variable stiffness control for parametric vibrations of cables. Earthquake Engineering and Engineering Vibration 5, 215-222 (2006)

15. Das, T., Mukherjee, R.: Optimally switched linear systems. Automatica 44, 1437-1441 (2006)

16. Abu-Hilal, M.: Dynamic response of a double Euler-Bernoulli beam due to a moving constant load. J. Sound Vib. 297, 477-491 (2006)

17. Pisarski, D., Bajer, C.I.: Semi-active control of $1 \mathrm{D}$ continuum vibrations under a travelling load. J. Sound Vib. 329(2), 140-149 (2010)

18. Pontryagin, L.S., Bolytanskii, V.G., Gamkrelidze, R.V., Mishchenko, E.F.: The Mathematical Theory of Optimal Processes. Fizmatgiz, Moscow (1961)

19. Mohler, R.R.: Bilinear Control Processes. Academic, New York (1973)

20. Elliott, D.: Bilinear control systems. Matrices in Action. Springer, Dordrecht (2009)

21. Hooke, R., Jeeves, T.A.: Direct search solution of numerical and statistical problems. J. ACM 8, 212-229 (1961) 